\title{
Enhancement of product selectivity via enzyme immobilization in sequential degradation reactions of polymeric substrates
}

\author{
S. Varga, F. Xavier Malcata
}

\begin{abstract}
The balance equations pertaining to the modelling of a slap-shaped bead containing immobilized enzyme uniformly distributed which catalyzes the sequential reactions of degradation of a polymeric substrate were written and analytically solved in dimensionless form. The effect of the Thiele modulus on the selectivity of consumption of each multimeric product was studied for a simple case. Whereas plain diffusional regime leads to lower selectivities than plain kinetic regime, improvements in selectivity of species $A_{i}$ relative to species $A_{i+1}$ may be obtained at the expense of higher Thiele moduli within a limited range when the diffusivity of $A_{i}$ is larger than that of $A_{i+1}$, or when the pseudo first order kinetic constant describing the rate of consumption of $A_{i}$ is lower than that of $A_{i+1}$.
\end{abstract}

\section{List of symbols}

$\begin{array}{ll}A_{i} & - \\ C_{i}^{*} & \mathrm{~mol} \cdot \mathrm{m}^{-3} \\ C_{i} & \mathrm{~mol} \cdot \mathrm{m}^{-3} \\ C_{i, 0} & \mathrm{~mol} \cdot \mathrm{m}^{-3} \\ C_{i, 0}^{*} & - \\ D_{a p, i} & \mathrm{~m}^{2} \cdot \mathrm{s}^{-1} \\ k_{i} & \mathrm{~s}^{-1} \\ K_{m, i} & \mathrm{~mol} \cdot \mathrm{m}^{-3} \\ L & \\ N & \mathrm{~m} \\ N & -\end{array}$

polymeric substrate containing $i$ monomeric subunits normalized counterpart of $C_{i}$ concentration of substrate $A_{i}$ initial concentration of substrate $A_{i}$ normalized counterpart of $C_{i, 0}$ apparent diffusivity of substrate $A_{i}$ pseudo-first order rate constant Michaelis-Menten constant associated with substrate $A_{i}$

half-thickness of the catalyst slab number of monomeric subunits of the largest substrate molecule

$\begin{array}{lll}\text { Th } & - & \text { Thiele modulus } \\ v_{i} & \mathrm{~mol} \cdot \mathrm{m}^{-3} \cdot \mathrm{s}^{-1} & \text { rate of rection of substrate } A_{i}\end{array}$ $\begin{array}{ll}v_{i} & \mathrm{~mol} \cdot \mathrm{m}^{-3} \cdot \mathrm{s}^{-1} \text { rate of rection of substrate } A_{i} \\ v_{\text {max }, i} & \mathrm{~mol} \cdot \mathrm{m}^{-3} \cdot \mathrm{s}^{-1} \text { maximum rate of reaction under }\end{array}$ saturating conditions of substrate $A_{i}$ longitudinal coordinate

$\begin{array}{ll}x & \text { m } \\ S_{i, i+1} & -\end{array}$

\section{S. Varga, F.X. Malcata}

Escola Superior de Biotecnologia, Universidade Católica Portuguesa, Rua Dr. António Bernardino de Almeida, 4200 Porto, Portugal

\section{Greek symbols \\ $\eta_{i} \quad-$ $\varpi_{i} \quad-$ \\ 1 Introduction} ratio of maximum rates of reaction ratio of apparent diffusivities

The use of enzymes for chemical degradation of polymeric products possesses advantages in what concerns environmental factors for a number of reasons: e.g. enzyme-catalyzed hydrolyses proceed at relatively high rates at room temperature and mild $\mathrm{pH}$ whereas the acid hydrolysis counterpart not only requires strong soluble acids (which must be neutralizated prior to disposal) but also requires considerable extents of energy (because acceptable rates are obtained at elevated temperatures). One good example of industrial application pertains to amylases acting upon starch: the action of $\alpha$ amylases reduces the solution viscosity by acting randomly along the glucose chain at the $\alpha-1,4$ glycosidic bonds ( $\alpha$ amylase is often called a starch-liquefying enzyme for this reason), whereas $\beta$-amylase can attack starch $\alpha-1,4$ bonds only on the nonreducing ends of the polymer chains and always produces maltose when a linear chain is hydrolyzed (because of the characteristic production of the sugar maltose, this enzyme is also called saccharifying enzyme); another example encompasses amyloglucosidase (also called glucoamylase) which attacks primarily the nonreducing $\alpha-1,4$ linkages at the ends of starch, glycogen, dextrins, and maltose [1]. Sequential treatment with $\alpha$-amylase and glucoamylase are utilized where pure glucose rather than maltose is desired (viz. in distilleries, as opposed to breweries, and in the manufacture of glucose corn syrups); other common applications of amylase preparations encompass breadmaking (in the production of fermentable saccharides needed for carbon dioxide production in leavening), fruit juice processing (to reduce turbidity due to insolubility of starch), papermaking (to liquefy starch coatings to a desired viscosity for application to fibers), in textile production (to strengthen warp threads before weaving or remove size from wooven material in order to promote uniform dyeing of fabric), and in candy manufacture (to reach the desired softness) [2].

Of particular interest here are the reactions effected by soluble exo-hydrolases (i.e. enzymes that cleave ester, glycosidic, or peptide bonds next to the ends of polymeric carbon backbones thus releasing monomeric subunits). If the feedstocks for these reactions are complex mixtures of linear biopolymers of various chain lengths, modelling of the reaction 
kinetics (and hence of reactor performance) becomes particularly cumbersome and therefore one often resorts to lumping together all chemical species with more than one monomeric subunit into a single imaginary species. Shortcomings of the aforementioned lumped approach have been made apparent previously for various reactor configurations $[3,4]$. One way to overcome these problems is to use multisubstrate Michaelis-Menten kinetics: since use of such rate expression in its integrated form may lead to difficulties in handling material balances to reactors due to its nonexplicit nature, approximations have been discussed which encompass transformation of the rate expression after assuming that the distribution of concentrations is a continuous rather than a discrete function [5] and explicitation of the concentration of each substrate with respect to time via a Taylor series methodology [6]. Theoretical work has been reported on the determination of the optimum operating time for batch isothermal performance of enzyme-catalyzed multisubstrate reactions [7], whereas the ratio of production of one given product to another given product in isothermal systems has been analyzed analytically for first order kinetics [8] and Langmuir-Hinshelwood kinetics $[9,10]$. However, virtually no information exists on multisubstrate reactions catalyzed by immobilized enzymes that may be useful for the predesign of reaction systems. It is thus the purpose of this work to shed some light into the uniqueness of the reaction systems performing multisubstrate reactions effected by immobilized enzymes, especially in terms of the changes in selectivity brought about by intraparticle concentration gradients.

\section{2}

\section{Theory}

Assume that an enzyme immobilized in a slab-shaped bead catalyzes the following set of elementary chemical reactions:

$A_{N} \stackrel{k_{N}}{\rightarrow} A_{N-1}+A$

$A_{N-\mathrm{I}} \stackrel{k_{N-1}}{\rightarrow} A_{N-2}+A$

$\ldots \ldots \ldots$

$A_{i} \stackrel{k_{i}}{\rightarrow} A_{i-1}+A$

........

$A_{2} \stackrel{k_{2}}{\rightarrow} A+A$

where the polymeric substrate containing $i$ monomeric subunits is denoted as $A_{i}(2 \leqslant i \leqslant N$, where $N$ is the number of monomeric subunits of the longest polymer under consideration). Assuming that (i) the rate of reactions is well described by a multisubstrate Michaelis-Menten mechanism and (ii) the monomeric unit does not virtually bind to the enzyme, then the rate expression reads [11]:

$$
v_{i}=\frac{\frac{v_{\max , i}}{K_{m, i}} C_{i}}{1+\sum_{j=2}^{N} \frac{C_{j}}{K_{m, j}}} ; \quad 2 \leqslant i \leqslant N
$$

where $v_{\text {max }, i}$ and $K_{m, i}$ denote the maximum rate (i.e., the rate under saturation conditions of substrate) and the MichaelisMenten parameter associated with the substrate containing $i$ monomeric units, respectively. Assuming, as usual, that the
Michaelis-Menten parameters of all substrates are approximately equal to one another (say, $K_{m}$ ), then Eq. (2) becomes:

$$
v_{i}=\frac{v_{\max , i} C_{i}}{K_{m}+\sum_{j=2}^{N} C_{j}} ; \quad 2 \leqslant i \leqslant N
$$

Given the stoichiometry of the reaction, Eq. (3) simplifies to:

$v_{i}=k_{i} C_{i} ; \quad 2 \leqslant i \leqslant N$

where the pseudo-first order rate constant is defined as:

$$
k_{i} \equiv \frac{v_{\max , i}}{K_{m}+\sum_{j=2}^{N} C_{j}}=\frac{v_{\max , i}}{K_{m}+\sum_{j=2}^{N} C_{j, 0}} ; \quad 2 \leqslant i \leqslant N
$$

and where subscript 0 denotes initial conditions.

The steady-state differential mass balances to the various polymeric substrates under the assumption that transport occurs only by molecular diffusion (and associated boundary conditions) are given by:

$$
\begin{aligned}
& D_{a p, N} \frac{d^{2} C_{N}}{d x^{2}}-k_{N} C_{N}=0, \\
& D_{a p, i} \frac{d^{2} C_{i}}{d x^{2}}-k_{i} C_{i}+k_{i+1} C_{i+1}=0 ; \quad 2 \leqslant i \leqslant N-1 \\
& \text { @ } x=0, \frac{d C_{i}}{d x}=0 ; \quad 2 \leqslant i \leqslant N \\
& \text { @) } x=L, C_{i}=C_{i, 0} ; \quad 2 \leqslant i \leqslant N
\end{aligned}
$$

where $x$ is the longitudinal coordinate, $D_{a p, i}$ and $C_{i, 0}$ the apparent diffusivity and the surface concentration of the polymeric substrate containing $i$ subunits, and $L$ the halfthickness of the slab. Equation (6) may be rewritten as:

$$
\begin{aligned}
& \frac{d^{2} C_{N}^{*}}{d x^{* 2}}-T h^{2} C_{N}^{*}=0, \\
& \frac{d^{2} C_{N-1}^{*}}{d x^{* 2}}-\frac{\eta_{N-1}}{\varpi_{N-1}} T h^{2} C_{N-1}^{*}+T h^{2} C_{N}^{*}=0, \\
& \frac{d^{2} C_{i}^{*}}{d x^{* 2}}-\frac{\eta_{i}}{\varpi_{i}} T h^{2} C_{i}^{*}+\frac{\eta_{i+1}}{\varpi_{i+1}} T h^{2} C_{i+1}^{*}=0 ; \quad 2 \leqslant i \leqslant N-2 \\
& @ x^{*}=0, \frac{d C_{i}^{*}}{d x^{*}}=0 ; \quad 2 \leqslant i \leqslant N \\
& @ x^{*}=1, C_{N}^{*}=1, \\
& @ x^{*}=1, C_{i}^{*}=C_{i, 0}^{*} ; \quad 2 \leqslant i \leqslant N-1
\end{aligned}
$$

where the normalized variables are given by:

$$
\begin{aligned}
C_{N}^{*} & \equiv \frac{C_{N}}{C_{N, 0}}, \\
C_{i}^{*} & \equiv \frac{C_{i}}{C_{N, 0}}, \\
C_{i, 0}^{*} & \equiv \frac{C_{i, 0}}{C_{N, 0}}, \\
x^{*} & \equiv \frac{x}{L},
\end{aligned}
$$


and where the dimensionless parameters are defined as:

$\varpi_{i} \equiv \frac{D_{a p, i}}{D_{a p, N}}$,

$\eta_{i} \equiv \frac{v_{\max , i}}{v_{\max , N}}$,

$T h^{2} \equiv \frac{L^{2} v_{\max , N}}{C_{N, 0} D_{a p, N}} ;$

Solution of the set of linear ordinary differential equations, denoted by Eq. (7), following sequential steps of solving the homogeneous equation and finding a particular integral yields, after some algebraic work, the following analytical expressions:

$C_{N}^{*}=\lambda_{0,0}^{*} \frac{\cosh \left\{T h x^{*}\right\}}{\cosh \{T h\}}$,

$C_{N-1}^{*}=\lambda_{1,1} \frac{\cosh \left\{\sqrt{\frac{\eta_{N-1}}{\varpi_{N-1}}} T h x^{*}\right\}}{\cosh \left\{\sqrt{\frac{\eta_{N-1}}{\varpi_{N-1}}} T h\right\}}+\lambda_{1,0} \frac{\cosh \left\{T h x^{*}\right\}}{\cosh \{T h\}}$,

$C_{N-i}^{*}=\lambda_{i, i} \frac{\cosh \left\{\sqrt{\frac{\eta_{N-i}}{\varpi_{N-i}}} T h x^{*}\right\}}{\cosh \left\{\sqrt{\frac{\eta_{N-i}}{\varpi_{N-i}}} T h\right\}}+\sum_{j=1}^{i-1} \lambda_{i, j} \frac{\cosh \left\{\sqrt{\frac{\eta_{N-j}}{\varpi_{N-j}}} T h x^{*}\right\}}{\cosh \left\{\sqrt{\frac{\eta_{N-j}}{\varpi_{N-j}}} T h\right\}}$

$$
+\lambda_{i, 0} \frac{\cosh \left\{T h x^{*}\right\}}{\cosh \{T h\}} ; \quad i=2,3, \ldots, N-3, N-2
$$

where the coefficients are defined by the following recurrence relations:

$\lambda_{0,0}^{*}=1$,

$\lambda_{1,1} \equiv C_{N-1,0}^{\star}+\frac{1}{1-\frac{\eta_{N-1}}{\varpi_{N-1}}}$,

$\lambda_{1,0} \equiv-\frac{1}{1-\frac{\eta_{N-1}}{\varpi_{N-1}}}$,

$\lambda_{i, i} \equiv C_{N-i, 0}^{*}+(-1)^{i+1} \frac{\prod_{k=1}^{i-1} \frac{\eta_{N-k}}{\varpi_{N-k}}}{\prod_{l=1}^{i}\left(1-\frac{\eta_{N-e}}{\varpi_{N-e}}\right)}+\sum_{m=1}^{i-1}(-1)^{i-1+m} \frac{\frac{\eta_{N-i+1}}{\varpi_{N-i+1}}}{\frac{\eta_{N-m}}{\varpi_{N-m}}-\frac{\eta_{N-i}}{\varpi_{N-i}}} \lambda_{i-1, m} ; \quad i=2,3, \ldots, N-3, N-2$ be defined as a type III selectivity [12], viz.:

$S_{i, i+1} \equiv \frac{D_{a p, i}\left(\frac{d C_{i}}{d x}\right)_{x=L}}{D_{a p, i+1}\left(\frac{d C_{i+1}}{d x}\right)_{x=L}}=\frac{\varpi_{i}\left(\frac{d C_{i}^{*}}{d x^{*}}\right)_{x^{*}=1}}{\varpi_{i+1}\left(\frac{d C_{i+1}^{*}}{d x^{*}}\right)_{x^{*}=1}}$

$i=2,3, \ldots, N-3, N-2$

In view of Eqs. (10) and (11), the first (and more meaningful) of
The sselectivity of each reaction relative to another may then

$$
\begin{aligned}
\lambda_{i, j} \equiv(-1)^{i+j} \frac{\frac{\eta_{N-i+1}}{\varpi_{N-i+1}}}{\frac{\eta_{N-i+1}}{\varpi_{N-i+1}}-\frac{\eta_{N-i}}{\varpi_{N-i}}} \lambda_{i-1, j} ; \quad i=2,3, \ldots, N-3, N-2, j=1,2, \ldots, i-2, i-1 \\
\lambda_{i, 0} \equiv(-1)^{i} \frac{\frac{\eta_{N-i+1}}{\bar{\sigma}_{N-i+1}}}{1-\frac{\eta_{N-i}}{\bar{w}_{N-i}}} \lambda_{i-1,0} ; \quad i=2,3, \ldots, N-3, N-2
\end{aligned}
$$




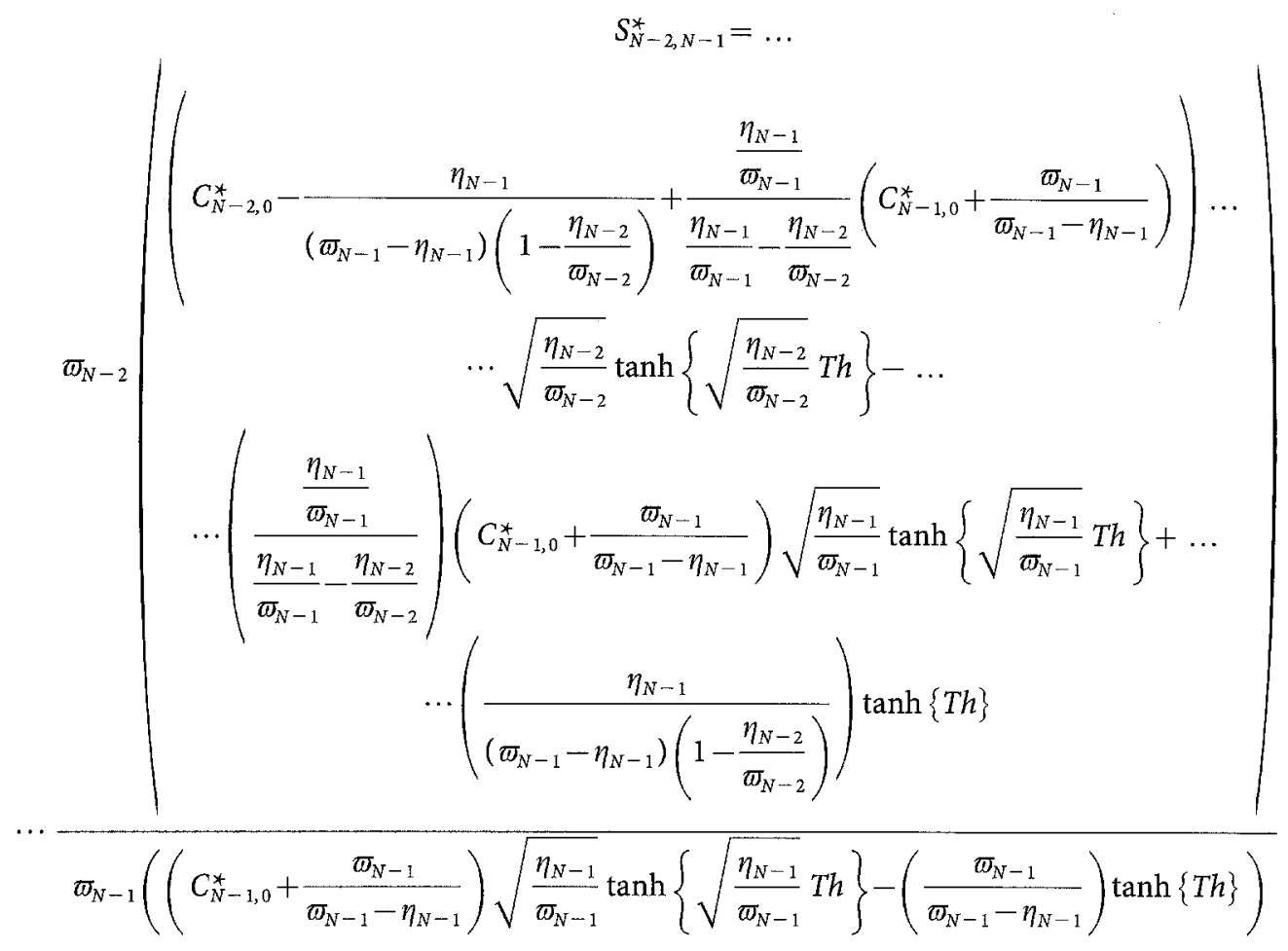

3

\section{Numerical example}

One efficient simple way of testing the effect of immobilization upon selectivity is to consider only the largest consecutive polymers in the degradation series (i.e., $A_{N-1}$ and $A_{N-2}$ ) with equal surface concentrations (say, $C_{N-1,0}^{*}=C_{N-2,0}^{*}=1$ ) and assume that either (i) $\eta_{N-1}=\eta_{N-2}=1$ (say) or (ii) $\varpi_{N-1}=\varpi_{N-2}=1$ (say). The first situation (i) yields: (or 0.99) and $\eta_{N-2}=0.90$ (or 0.98), and for $\eta_{N-1}=1.02$ (or 1.05) and $\eta_{N-2}=1.01$ (or 1.10).

\section{4}

Discussion and conclusions

It should be emphasized that the way selectivity was defined by Eq. (12) implies that both $A_{i}$ and $A_{i+1}$ are either consumed or

$S_{N-2, N-1}^{*}=\frac{\left(\begin{array}{c}\left(\varpi_{N-2}-\frac{\varpi_{N-2}^{2}}{\left(\varpi_{N-1}-1\right)\left(\varpi_{N-2}-1\right)}+\frac{\varpi_{N-2}}{\varpi_{N-2}-\varpi_{N-1}}\left(1+\frac{\varpi_{N-1}}{\varpi_{N-1}-1}\right)\right) \sqrt{\varpi_{N-2}} \tanh \left\{\sqrt{\frac{1}{\varpi_{N-2}}} T h\right\}-\ldots \\ \cdots\left(\frac{\varpi_{N-2}^{2}}{\varpi_{N-2}-\varpi_{N-1}}\right)\left(1+\frac{\varpi_{N-1}}{\varpi_{N-1}-1}\right) \sqrt{\frac{1}{\varpi_{N-1}}} \tanh \left\{\sqrt{\frac{1}{\varpi_{N-1}}} T h\right\}+\left(\frac{\varpi_{N-2}^{2}}{\left(\varpi_{N-1}-1\right)\left(\varpi_{N-2}-1\right)}\right) \tanh \{T h\}\end{array},\right.}{\left(1+\frac{\varpi_{N-1}}{\varpi_{N-1}-1}\right) \sqrt{\varpi_{N-1}} \tanh \left\{\sqrt{\frac{1}{\varpi_{N-1}}} T h\right\}-\left(\frac{\varpi_{N-1}^{2}}{\varpi_{N-1}-1}\right) \tanh \{T h\}}$,

whereas the second situation (ii) yields:

$S_{N-2, N-1}^{*}=\frac{\left(\begin{array}{l}\left(1-\frac{\eta_{N-1}}{\left(1-\eta_{N-1}\right)\left(1-\eta_{N-2}\right)}+\frac{\eta_{N-1}}{\eta_{N-1}-\eta_{N-2}}\left(1+\frac{1}{1-\eta_{N-1}}\right)\right) \sqrt{\eta_{N-2}} \tanh \left\{\sqrt{\eta_{N-2}} T h\right\}-\cdots \\ \cdots\left(\frac{\eta_{N-1}}{\eta_{N-1}-\eta_{N-2}}\right)\left(1+\frac{1}{1-\eta_{N-1}}\right) \sqrt{\eta_{N-1}} \tanh \left\{\sqrt{\eta_{N-1}} T h\right\}+\left(\frac{\eta_{N-1}}{\left(1-\eta_{N-1}\right)\left(1-\eta_{N-2}\right)}\right) \tanh \{T h\}\end{array}\right)}{\left(1+\frac{1}{1-\eta_{N-1}}\right) \sqrt{\eta_{N-1}} \tanh \left\{\sqrt{\eta_{N-1}} T h\right\}-\left(\frac{1}{1-\eta_{N-1}}\right) \tanh \{T h\}}$

Equation (15) is graphically plotted in Fig. 1 as a function of Th for ${\sigma_{N-1}}_{N}=0.95$ (or 0.99) and $\varpi_{N-2}=0.90$ (or 0.98 ), and for $\varpi_{N-1}=1.02$ (or 1.05) and ${\sigma_{N-2}}=1.01$ (or 1.10). Equation (14) is graphically plotted in Fig. 2 as a function of Th for $\eta_{N-1}=0.95$ produced, which should (in principle) give positive values for $S_{i, i+1}$. However, since it is assumed that the concentrations of all types of polymeric species on the surface of the catalyst are not nil, then situations where species $A_{i}$ is net-consumed and 

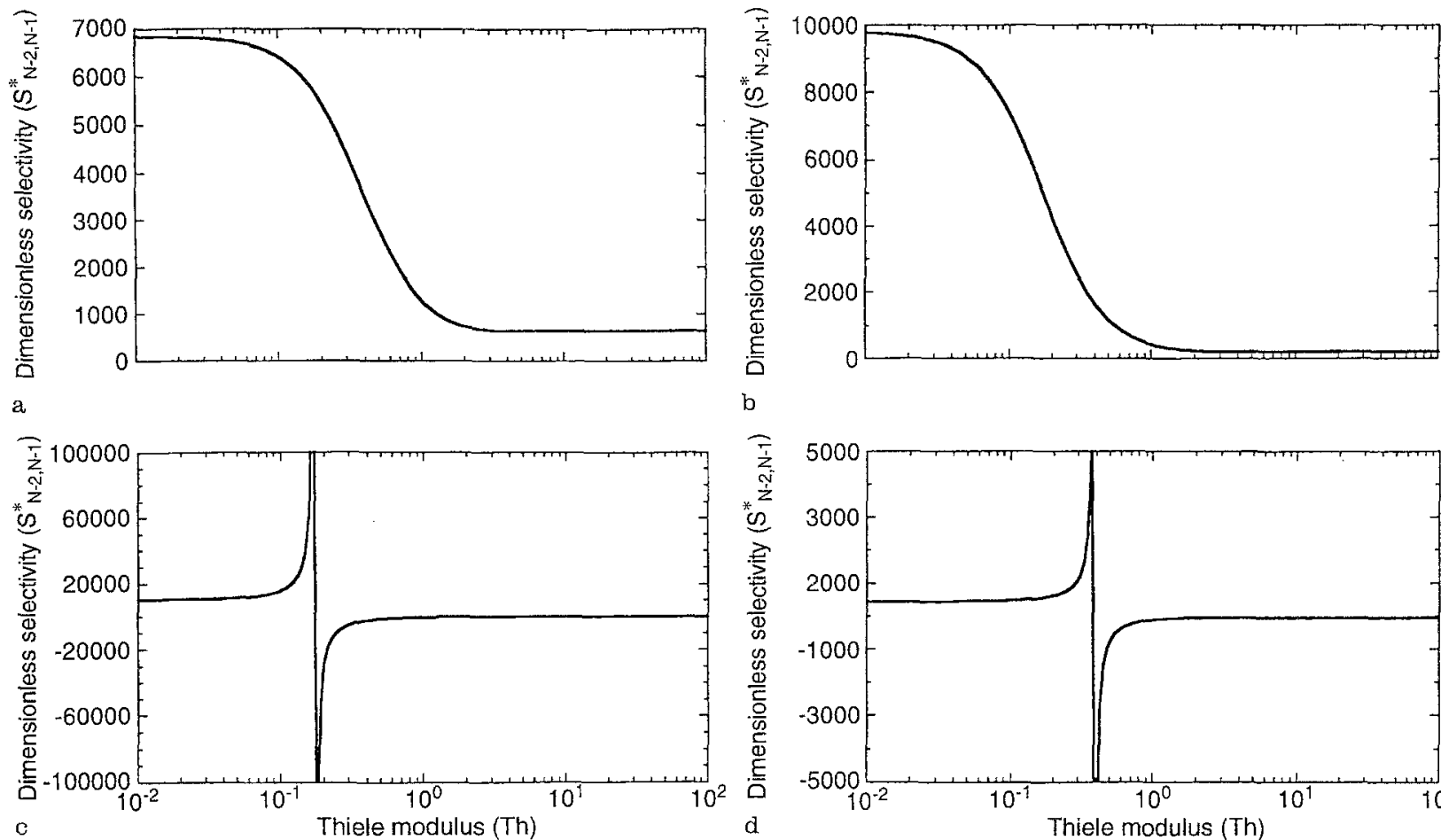

Fig. 1a-d. Plot of $S_{N-2, N-1}^{*}$ versus $T h$ for. a $\varpi_{N-1}=0.95$ and $\varpi_{N-2}=0.90, \mathbf{b} \varpi_{N-1}=0.99$ and $\varpi_{N-2}=0.98, \mathbf{c} \varpi_{N-1}=1.02$ and $\varpi_{N-2}=1.01, \mathbf{d} \varpi_{N-1}=1.05$ and $\bar{\omega}_{N-2}=1.10$

species $A_{i+1}$ is net-formed, or vice versa will give rise to negative values for $S_{i, i+1}$; one such situation is depicted in Fig. $2 \mathrm{c}-\mathrm{d}$. Furthermore, it is interesting to note that a sudden alteration of the form of the concentration gradients within the porous catalyst (which should then lead to an asymptotic change from a positive to a negative value of $S_{i, i+1}$ ) may occur at a given value of $T h$ when the diffusivity of each product molecule is higher than the diffusivity of its reactant molecule (see Fig. 1c-d) or when the rate of consumption of each product molecule is lower than the rate of consumption of its reactant molecule (see Fig. $2 \mathrm{a}-\mathrm{b}$ ); in the former situation the critical Thiele modulus increases with increasing values for the ratio $\varpi_{i+1} / \varpi_{i}$ (see Fig. $1 \mathrm{c}-\mathrm{d}$ ) whereas in the latter situation the critical Thiele modulus decreases with increasing values for the ratio $\bar{\omega}_{i+1} / \bar{\omega}_{i}$ (see Fig. $2 \mathrm{a}-\mathrm{b}$ ).

When a set of two consecutive reactions (as is the case of conversion of $A_{N}$ into $A_{N-1}$ followed by conversion of $A_{N-1}$ into $A_{N-2}$ ) take place within a porous solid catalyst the concentrations of the initial reactant (i.e. $A_{N}$ ) and of the intermediate product (i.e. $A_{N-1}$ ) are significantly different from those prevailing on the bulk of the outer fluid or even on the surface of the catalyst. The intermediate product molecules formed within the pore structure have a high probability of reacting further before they can diffuse out of the pore; consequently catalysts with narrow pores (i.e. at high $T h$ values) should give lower yields of the intermediate product than those with large pores (i.e. at low $T h$ values) if no differences existed between the diffusivities of said compounds [13]. This rationale may account for the general increasing behaviour of the first portion of the curves representing $S_{N-2, N-1}^{*}$ vs. Th as apparent in Figs. $1 c-d$ and $2 a-b$. However, if the diffusivities of the species in question are different from one another, the behavior of the selectivity with respect to the Thiele modulus may be completely reversed. This is the case illustrated in Fig. $1 \mathrm{a}-\mathrm{b}$ where the rates of consumption of the species in question were deliberately set equal to one anoter but the diffusivity of $A_{N-2}$ is below that of $A_{N-1}$, and the diffusivity of $A_{N-1}$ is below that of $A_{N}$; in this situation, the higher difficulty of each product to leave the porous catalyst than the corresponding reactant makes it more susceptible to consumption by chemical reaction.

Irrespective of the pattern of variation of $S_{i, i+1}$ with $T h$, the range of $T h$ where the most abrupt behavior is observed is typically $0.1-1$; this range is essentially the same irrespective of the relation between the value for $D_{a p}$ and $v_{\max }$ for the species considered. The limit of the behaviour of $S_{N-2, N-1}^{*}$ when $T h$ tends to zero corresponds to the situation where no diffusional limitations exist with respect to any substrate; in this case, the slab behaves as a well-stirred system containing free enzyme. It is apparent from inspection of Figs. $1 \mathrm{c}-\mathrm{d}$ and $2 \mathrm{a}-\mathrm{b}$ that existence of diffusional limitations does, up to some threshold, improve the selectivity of the immobilized enzyme. This conclusion is in disagreement with the general assertion that intraparticle diffusional limitations, under isothermal or nonisothermal conditions, reduce the selectivity below that which can be achieved in their absence [13]; however, such general assertion always holds when the purely diffusional regime (i.e. when Th tends to infinity) is compared with the purely kinetic regime as can be easily concluded from inspection of Figs. 1 and 2. 

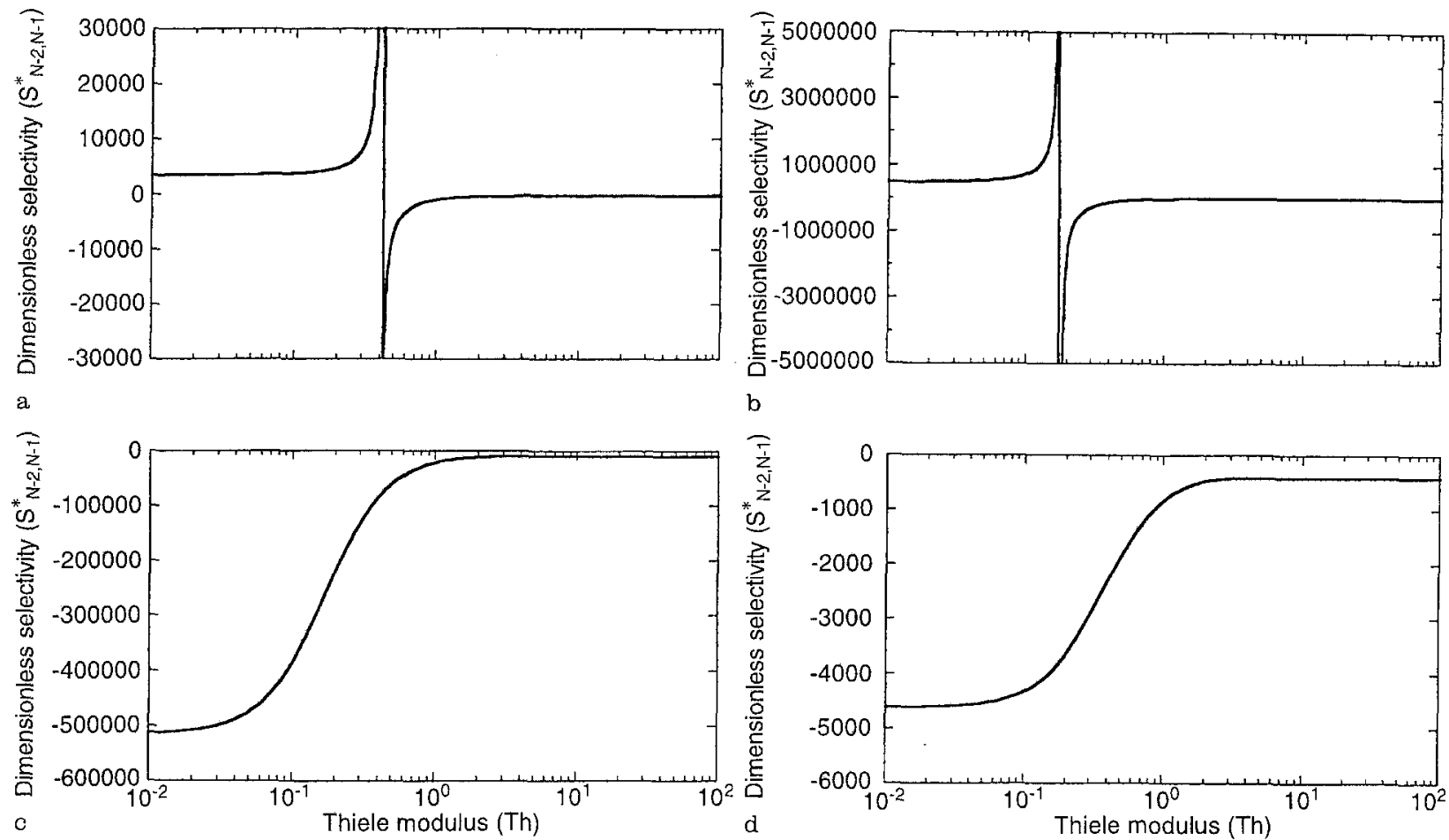

Fig. 2. Plot of $S_{N-2, N-1}^{*}$ versus $T h$ for. a $\eta_{N-1}=0.95$ and $\eta_{N-2}=0.90, \mathbf{b} \eta_{N-1}=0.99$ and $\eta_{N-2}=0.98, \mathbf{c} \eta_{N-1}=1.02$ and $\eta_{N-2}=1.01$, d $\eta_{N-1}=1.05$ and $\eta_{N-2}=1.10$

\section{References}

1. Bailey, J.E.; Ollis, D.F.: Biochemical Engineering Fundamentals, McGraw-Hill, New York (1986) 163-164

2. Weiser, H.H.: Practical Food Microbiology and Technology, p. 37, AVI, Westport (1962)

3. Malcata, F.X.: J. Chem. Educ. 68 (1991) 288

4. Malcata, F.X.: Int. J. Math. Educ. Sci. Technol. 24 (1993) 27

5. Silva, T.R.; Malcata, F.X.: Biopr. Eng. 10 (1994) 235

6. Costa, R.; Malcata, F.X.: Biopr. Eng. 10 (1994) 155

7. Malcata, F.X.: Biopr. Eng. 10 (1994) 173
8. Smith, J.M.: Chemical Engineering Kinetics, McGraw-Hill, New York (1981) 507-511

9. Roberts, G.; Satterfield, C.N.: Ind. Eng. Chem. Fund. Quart. 4 (1965) 288

10. Hutchings, J.; Carberry, J.J.: AIChE J. 12 (1966) 20

11. Segel, I.H.: Enzyme Kinetics, Wiley, New York (1975) 113-118

12. Wheeler, A.; in Frankenburg, W.G.; Komarewsky, V.I., \& Rideal, E.K. (eds.): Advances in Catalysis, Academic Press, New York (1951) 313

13. Hill, C.G.: An Introduction to Chemical Engineering Kinetics and Reactor Design, Wiley, New York (1977) 468-474 